\title{
La producción sobre documentación audiovisual y archivos televisivos en la Web of Science (2000-2016)
}

The production on audiovisual documentation and television archives in the Web of Science (2000-2016)

Rocío Gómez-Crisóstomo, Luz Ma Romo-Fernández, Jorge Caldera-SerRano

Facultad de Ciencias de la Documentación y la Comunicación, Universidad de Extremadura. Plazuela de Ibn Marwan, s/n. 06071 , Badajoz,mrgomcri@unex.es, Imromfer@unex.es, jcalser@unex.es

\begin{abstract}
Resumen
Se analiza la producción científica contenida en la Web of Science en el periodo 2000-2016 sobre documentación audiovisual y archivos televisivos, publicada en fuentes indizadas bajo la categoría de Information Science \& Library Science. Tras realizar las consultas, y establecer un filtro para eliminar aquellos no considerados relevantes, se obtuvo un total de 87 trabajos, que son estudiados para analizar el número anual de documentos, número anual de citas recibidas, tipología documental, idioma de publicación, fuentes en las que se difunden, países, instituciones y autores involucrados en dichas publicaciones, así como los patrones de coautoría seguidos por dichos autores.
\end{abstract}

Palabras clave: Archivos de televisión. Documentación audiovisual. Producción científica. Investigación. Web of Science.

\section{Introducción}

La Ciencia de la Documentación es entendida como una ciencia transversal, una ciencia auxiliar de otras disciplinas, disciplinas que le dan sentido y en cierta manera le otorgan el contexto en el cual poder implantarse, crecer y ayudar. Un ejemplo de dicha adaptación de las Ciencias de la Documentación a una disciplina concreta serían los trabajos y las técnicas desarrolladas e implantadas en los medios de comunicación, para nuestro estudio, específicamente en las cadenas de Televisión.

La presencia de los Servicios de Documentación y la Televisión no siempre ha sido fácil. Con la estandarización de las emisiones televisivas en la década de los 50 del siglo pasado, no llegó la presencia de la gestión documental a estas instituciones (en el caso español), debiendo esperar a la década de los 70 para ver las primeras labores serias en el ámbito documental, de la mano de bibliotecarios que hacían grandes esfuerzos por extrapolar técnicas bibliográficas a formatos diversos con usuarios y requerimientos muy alejados a los de la biblioteca. No obstante, esto

\begin{abstract}
The scientific production contained in Web of Science in the period 2000-2016 on audiovisual documentation and television archives, published in sources indexed under the category of Information Science \& Library Science, is analyzed. After completing the relevant queries and establishing a filter to eliminate those works not considered relevant, a total of 87 works were extracted, analysing the annual number of documents, annual number of citations received, document type, language of publication, sources in which the works are published, countries, institutions and authors involved in such publications, as well as the co-authorship patterns followed by those authors.

Keywords: Television archives. Audiovisual documentation. Scientific production. Research. Web of Science.
\end{abstract}

fue modificándose, de tal manera que la aparición de las cadenas autonómicas y las posteriores televisiones privadas, venían aparejadas con la presencia en sus sistemas audiovisuales de los servicios de documentación. Evidentemente no siempre tuvieron la misma presencia, y no se les otorgó la misma importancia (lo que llega hasta nuestros días) pero desde hace muchos años (década de los 80 del siglo pasado) es impensable comenzar las emisiones de una cadena de televisión, sin contar con una estructura documental fija. Esta realidad es pareja a la de otros países desarrollados, aunque hemos de señalar que la importancia dada en el ámbito anglosajón ha sido mayor que la aportada en otras zonas del planeta.

El concepto de audiovisual no siempre ha estado claro. La escasez de investigaciones existentes antes de mediados de los 90 , hizo en cierta manera necesario tratar qué se entendía por "audiovisual" desde un punto de vista integrador: documentación cinematográfica (folletos, cartelería, el propio material fílmico, etc.), documentación sonora y/o radiofónica, documen- 
tación fotográfica y/o fotoperiodística, documentación televisiva... Todo era entendido en el amplio concepto de lo audiovisual. Se indica anteriormente que era comprensible, ya que los pocos trabajos e investigadores analizan en muchos casos esta información de manera general. Posteriormente, estos conceptos se han ido aclarando, y el de audiovisual ha quedado plasmado como aquellos documentos que cuentan con información audiovisual, sonora y de imagen en movimiento, con relación sincrónica o asíncrona. Pero esta definición de lo audiovisual nos lleva a una pregunta, ¿existe material no audiovisual que podría ser material útil para televisión? Evidentemente, se integra en el objeto de estudio de la documentación televisiva material que no es propiamente audiovisual, pero que tiene pleno sentido que esté presente en estos departamentos, tales como material captado previo a la existencia del magnetófono, o simplemente que no se ha captado el material sonoro por errores técnicos o por requerimientos legales (Póveda, 2010).

Los servicios de gestión documental de las cadenas televisivas han evolucionado hacia los modernos Media Asset Management (o Digital Asset Management). Independientemente de su evolución, el porqué de estos servicios de documentación viene de la mano de la necesidad que tiene el periodista de material para generar nuevos productos. ¿Podría emitir una cadena sin archivo televisivo? Sin duda que sí, cuestión aparte sería la calidad de dichos productos, especialmente en el ámbito informativo. Los departamentos existen porque los usuarios lo necesitan, los periodistas requieren recursos audiovisuales ante la imposibilidad de captar todo el material que necesitan. Por lo tanto, tienen una rentabilidad relacionada con la credibilidad y reputación de la cadena, pero muy especialmente con el abaratamiento de los costos de producción. Los departamentos de documentación existen porque son rentables. Tal es su importancia en la actualidad, que algunos centros cuentan los departamentos de documentación al mismo nivel que los recursos económicos y/o los recursos humanos, pero sobre todo son tenidos en cuenta como el mayor recurso patrimonial de la cadena.

Dentro del ámbito de la Documentación no es una línea de investigación especialmente tratada, aunque existen trabajos que lo han estudiado desde una visión general (Unesco, 1980; Caldera y Arranz, 2012; Saavedra, 2011; Edmonson, 2004; López, 2014; Hidalgo, 2013), análisis sobre el estado de los medios (Inarejos y Guallar, 2015); digitalización y prospección de futuro (Aguirreazaldegui, 2007; Caldera, 2008), selección (Giménez, 2007), específico de cine (López Yepes, 1992 y 2014), etc. Además, son especialmente interesantes los artículos aportados por parte de los profesionales de los medios (López de Quintana, 2007; Bustos, 2007; Hidalgo, 2005) y algunos aportados por profesionales junto con académicos (Caldera y Arranz, 2012; De la Cuadra y López, 2013).

Se estima que existe un número importante de estudios en el ámbito de la documentación audiovisual televisiva, tal y como se observa en un artículo realizado por Castillo y Soler (2014), artículo en el que se estudian diferentes bases de datos para analizar el número de artículos, tipo de documentos, fuentes, clasificación temática, autoría, etc. Sólo para autores españoles y con términos muy concretos, que han sido ampliados en este estudio.

Esta investigación quiere profundizar en la internalización y visualización de los trabajos en una de las principales bases de datos consultada por investigadores, como es la Web of Science, la cual hace un vaciado de los artículos de las principales revistas atendiendo al índice de impacto de las mismas.

\section{Metodología}

Para llevar a cabo este estudio, en primer lugar, se procedió a realizar una serie de consultas en la base de datos Web of Science para identificar aquellas publicaciones pertenecientes a revistas de la categoría Information Science \& Library Science, búsqueda que fue limitada al periodo de tiempo 2000-2016. El rango de fechas entre el que se ha realizado la consulta ha sido entre el 6 y 12 de febrero de 2017.

Una vez obtenidos los resultados (193.802 registros), éstos fueron sometidos a un filtro temático para seleccionar sólo aquellos que versasen sobre materias relacionadas con la televisión y el sector audiovisual, utilizando para ello consultas tanto con dichos términos, en su versión inglesa, como con otros del mismo ámbito, entre ellos, documentation, information, Media Asset Management, Digital Asset Management, etc.

Tras realizar todas las consultas pertinentes, se seleccionaron un total de 344 registros, que fueron analizados por un experto en la materia, para eliminar aquellos que produjesen ruido documental, es decir, que no fuesen realmente relevantes para el presente estudio. De este modo, el número de registros se redujo a 87 .

Estos 87 registros fueron incorporados a una base de datos diseñada ad-hoc, en la que posteriormente fueron realizadas las consultas necesarias para obtener resultados tales como el 
número de documentos por año y país, tipología documental e idioma de las publicaciones, tipo de autoría (sencilla o colectiva), número de citas recibidas por dichas publicaciones, revistas en las que se publican los documentos analizados, así como las instituciones que más documentos aglutinan en estas materias en el periodo estudiado. Todo ello, se muestra con mayor detalle en el apartado de resultados.

\section{Resultados}

En el gráfico 1 se observa el número de trabajos repartido a lo largo de los años. Se aprecia cómo no se encuentran documentos disponibles hasta el año 2002. (Se recuerda que la consulta se ha realizado incluyendo los años 2000 y 2001, en los cuáles no se han detectado resultados.) El total de documentos ha sido de 87 una vez se ha llevado a cabo el filtrado atendiendo a los criterios de búsqueda marcados en el apartado de metodología. El número de 87 es significativamente menor al total que nos devuelve la WoS, derivado de que existen muchos trabajos indexados con el término "audiovisual" y están relacionados con técnicas médicas, por lo que no tiene relación con la documentación audiovisual, los archivos de televisión, ni su gestión en las instituciones televisivas.

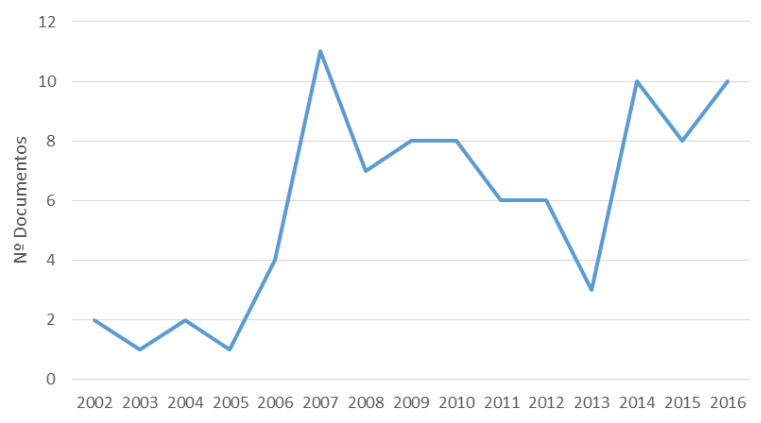

Gráfico 1. Número de documentos por año

Se estima que el número total no es elevado, por lo que entendemos que falta hoy en día una masa crítica de investigaciones en dicha materia, habida cuenta del escaso porcentaje resultante del total de la categoría analizada. Además, existen otras cuestiones que llevan a pensar que el número total de trabajos es escaso, que se analizarán cuando se estudien las fuentes de publicación, por lo que se podrá entender que existe una clara aglomeración de investigaciones en torno a una única publicación.

No es hasta el año 2005 cuando se aprecia un número "significativo", siendo el año pico el 2007, con un total de 11 trabajos publicados. 2014 y 2016 muestran una tendencia que pare- ce mantenerse en torno a los diez (señalar que no se recogen todas las investigaciones publicadas aún en el 2016). Cabe señalar igualmente que la WoS, en su política de expansión y de inclusión de otras bases de datos con el fin de aumentar el número de revistas, ha incorporado las diferentes revistas de Scielo así como ha integrado la base de datos Emerging Source Citation Index (ESCl), haciendo visibles aquellas revistas que están siendo analizadas por la WoS para ser incorporadas al JCR. De momento, al menos, el número de nuevas revistas es significativo. Sólo el ESCl supone prácticamente 5.000 nuevos títulos que, aunque no estén presentes en JCR, sí que incorporan los artículos y las citas de estas publicaciones. Cuantitativamente el cambio es muy significativo, ya que el número de trabajos es muy superior; $y$, por lo tanto, las posibilidades de obtener mayor número de resultados aumentan. Se integran un número importante de revistas en nuestra disciplina, algunas tan relevantes en el ámbito de lo audiovisual como las presentes en el registro de publicaciones de la Universidad Complutense, las cuáles, al estar muy relacionadas con la Facultad de Ciencias de la Información (Comunicación Social), cuentan con revistas con tendencia a publicar trabajos del ámbito de la documentación relacionados con las Ciencias de la Comunicación.

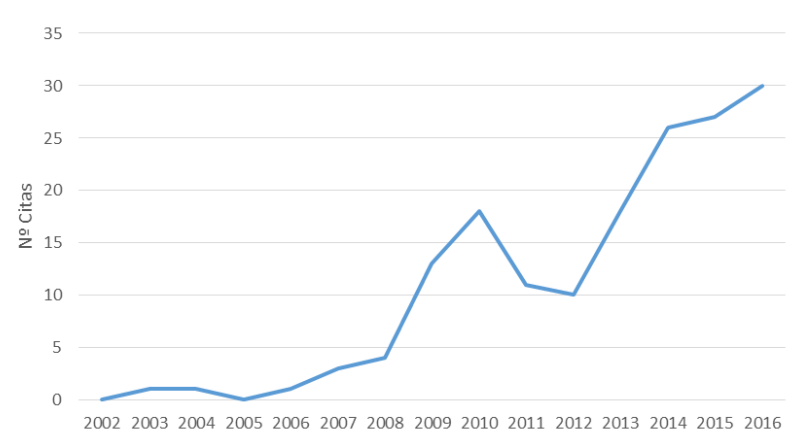

Gráfico 2. Número de citas por año

El gráfico 2 analiza el número de citas recibidas a lo largo de los años estudiados. El número total de citas recibidas es de 163 , número escaso para el total de trabajos (87). Esto genera una media de 1,87 citas por publicación. Esto da indicios del escaso número de investigadores (como después se analiza) y la escasa visibilidad, y seguramente internacionalización, de los resultados. Si se entiende la documentación aplicada a las Ciencias de la Información como una subárea, los investigadores en archivos de televisión son un porcentaje menor, aunque significativo. Por lo que se puede llegar a con- 
cluir la escasa importancia que la temática tiene en la representación tomada de la WoS.

No obstante, a la vista del gráfico 2 , se puede afirmar que existe un claro repunte, al ir subiendo de forma paulatina los trabajos con citas en la WoS. En el año 2016, aún sin cerrar en la WoS, se cuenta con un número significativo (30 citas), lo cual puede tener relación con la inclusión de la revista Cuadernos de Documentación Multimedia, especializada y específica en la gestión documental en los medios de comunicación. Otro hecho que marca el aumento en el número de trabajos y citas, es la inclusión de monográficos sobre Documentación audiovisual y Documentación en medios, realizado por la revista El Profesional de la Información, lo que hace también que exista un mayor número de publicaciones en años concretos, y, por lo tanto, aumento de las citas.

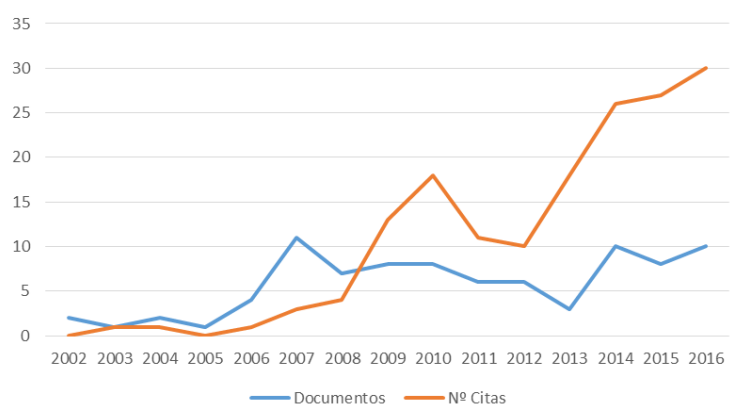

Gráfico 3. Comparación entre el número de documentos y de citas por año

No obstante, el gráfico 3 muestra que, aunque parece difícil superar la barrera de las diez publicaciones al año, sí que está siendo mucho mayor el número de citas recibidas por parte de estos trabajos. Se estima que se deben analizar más años para identificar si nos encontramos con una tendencia o simplemente un repunte en la citación por motivos que pueden ir a una mayor visibilidad de las publicaciones al estar presentes en la WoS o por la inclusión de revistas específicas tanto en la categoría analizada, como en la categoría de Communication.

Atendiendo a lo señalado con anterioridad, la presencia de dos monográficos de una revista española y de la inclusión de una nueva revista específica en la WoS desde el año 2015 (Documentación de las Ciencias de la Información también ha entrado en la WoS al igual que Cuadernos de Documentación Multimedia por la inclusión de la base de datos $\mathrm{ESCl}$, pero no está inscrita en la categoría analizada, sino en la de Communication), hace que nuestro país se presente en los trabajos indexados en la WoS como la gran potencia en esta materia.

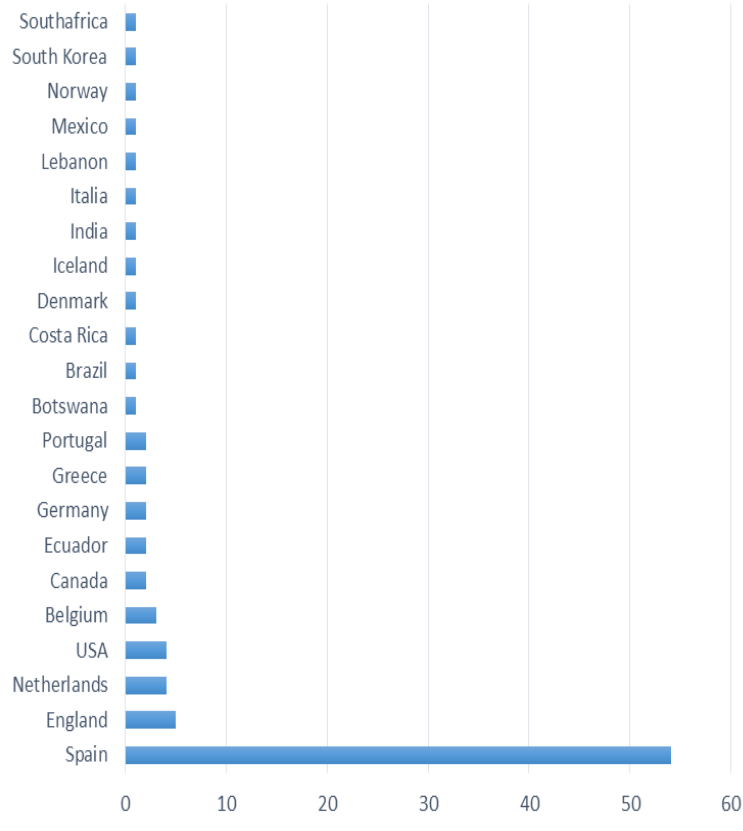

Gráfico 4. Número de documentos por país

Se recuperan un total de 54 publicaciones en los que al menos uno de sus autores está afiliado a una institución española. Esto hace que prácticamente un tercio de los artículos (el 62,06 $\%$ ) cuente con autores españoles. Inglaterra cuenta con cinco publicaciones, Estados Unidos con cuatro al igual que Holanda, los cuales cuentan con instituciones potentes (televisiones e instituciones) que trabajan sobre televisión. No obstante, son escasos el número de trabajos por parte de estos países. Para el resto de países el número es poco significativo, contando con dos o menos publicaciones.

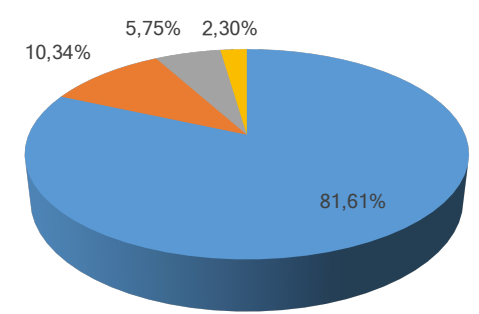

artículos $₫$ Actas de congresos $\square$ Revisiones de libros $₫$ Material editorial

Gráfico 5. Tipos de documentos

Respecto a los tipos documentales recuperados, claramente se observa que el artículo es el tipo documental más relevante, con un total de $81,61 \%$, lo que supone 71 de las publicaciones. Los trabajos presentados a congresos suponen 
un $10,34 \%$ de las publicaciones, con un total de 9 materiales publicados en las actas. Revisiones de libros junto con el epígrafe "Material editorial" son realmente residuales como número en nuestro trabajo.

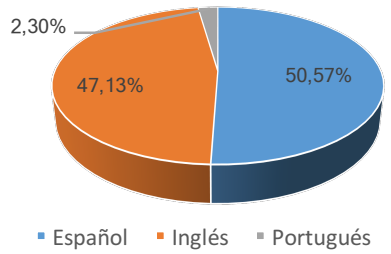

\section{Gráfico 6. Idiomas de las publicaciones}

El gráfico 6 aporta los diferentes idiomas en los que han sido publicados los trabajos. Era de esperar, a la vista de otros datos tales como autores y fuentes de procedencia, que estos datos dieran los siguientes resultados. Más de la mitad de las publicaciones son en español $(50,57 \%$, lo que se traduce en 44 publicaciones), lo cual no deja de ser una anomalía en la WoS habida cuenta que el mayor número de trabajos presentes en esta base de datos es en lengua inglesa, con una clara diferencia. No obstante, el $47,13 \%$ son escritos en inglés (41 publicaciones), lo que nos hace suponer que autores españoles no sólo publican en idioma español, sino que también publican en otras lenguas.

El alto porcentaje de trabajos en español junto con el análisis de autores nos lleva a pensar que existe un grupo importante de autores en España que trabajan en esta materia, pero de manera muy local, sin buscar -0 al menos sin lograr- la visibilidad y la internacionalización que se logra por medio de la publicación en inglés (lo que se observa del escaso número de citas), idioma de la ciencia en la actualidad.

La tabla I representa las fuentes en las que han sido publicados. El Profesional de la Información es, con diferencia, la revista más destacada, con un total de 30 aportaciones, focalizados en sus dos monográficos (aunque se encuentran igualmente publicaciones aisladas, pero en mucha menor cuantía). Basta con señalar que, de aquellas fuentes que cuentan con más de un trabajo, que suman un total de 62,39 de ellos corresponden a revistas españolas (El Profesional de la Información, Cuadernos de Documentación Multimedia y Revista Española de Documentación Científica), y si a este número se suma la revista Investigación Bibliotecológica, se aprecia que 45 aportaciones (de los 62 de las presentes en las revistas con más de dos publicaciones) son en español. Por lo tanto, la lectu- ra de esta información aporta un dato revelado con anterioridad: el núcleo de autores españoles es el más activo, o al menos, con mayor presencia en la WoS.

\begin{tabular}{|c|c|}
\hline Fuente & $N .^{\circ}$ \\
\hline Profesional de la Información & 30 \\
\hline Aslib Proceedings & 6 \\
\hline Investigación Bibliotecológica & 6 \\
\hline Cuadernos de Documentación Multimedia & 5 \\
\hline Journal of Librarianship and Information Science & 4 \\
\hline Revista Española de Documentación Científica & 4 \\
\hline Electronic Library & 3 \\
\hline Online Information Review & 2 \\
\hline Telematics and Informatics & 2 \\
\hline $\begin{array}{l}2015 \text { Digital Heritage International Congress Vol } 2 \\
\text { Analysis Interpretation Theory Methodologies Preservation } \\
\text { Standards Digital Heritage Projects Applications }\end{array}$ & 1 \\
\hline $\begin{array}{l}\text { Advancing Knowledge Expanding Horizons for Information } \\
\text { Science }\end{array}$ & 1 \\
\hline $\begin{array}{l}\text { African Journal of Library Archives and Information } \\
\text { Science }\end{array}$ & 1 \\
\hline Archiving 2006 Final Program and Proceedings & 1 \\
\hline Archiving 2008 Final Program and Proceedings & 1 \\
\hline $\begin{array}{l}\text { Archiving } 2011 \text { Preservation Strategies and Imaging } \\
\text { Technologies for Cultural Heritage Institutions and } \\
\text { Memory Organizations }\end{array}$ & 1 \\
\hline $\begin{array}{l}\text { Asian Digital Libraries Looking Back } 10 \text { Years and Forging } \\
\text { New Frontiers Proceedings }\end{array}$ & 1 \\
\hline $\begin{array}{l}\text { Biblios Revista de Bibliotecología y Ciencias de la } \\
\text { Información }\end{array}$ & 1 \\
\hline Informacao Sociedade Estudos & 1 \\
\hline Information Society & 1 \\
\hline Journal of Documentation & 1 \\
\hline Journal of Information Science & 1 \\
\hline $\begin{array}{l}\text { Journal of the American Society for Information Science } \\
\text { and Technology }\end{array}$ & 1 \\
\hline Knowledge Organization & 1 \\
\hline Library Collections Acquisitions Technical Services & 1 \\
\hline Library Hi Tech & 1 \\
\hline Library Trends & 1 \\
\hline Libri & 1 \\
\hline Mis Quarterly & 1 \\
\hline Multimedia for Cultural Heritage & 1 \\
\hline Program Electronic Library and Information Systems & 1 \\
\hline Research and Advanced Technology for Digital Libraries & 1 \\
\hline $\begin{array}{l}\text { Research and Advanced Technology for Digital Libraries } \\
\text { Proceedings }\end{array}$ & 1 \\
\hline $\begin{array}{l}\text { Research and Advanced Technology for Digital Libraries } \\
\text { Tpdl } 2016\end{array}$ & 1 \\
\hline Transinformaçao & 1 \\
\hline
\end{tabular}

Tabla I. Revistas/números de trabajos en la WoS

Analizar el gráfico 7 aporta un dato muy revelador: la autoría en solitario sigue siendo práctica habitual entre los investigadores en esta disciplina (más del $40 \%$ ). Un resultado realmente elevado en un ámbito de las Ciencias Sociales, donde los niveles de coautoría van creciendo. La coautoría entre dos autores supone tan sólo 
31 de los 87 trabajos analizados (cerca del 36 $\%$ ), mientras que, con más de tres autores, se encuentran tan sólo 21 (24,14 \%) (bajando drásticamente el número de aportaciones en colaboración con más de 4 autores).

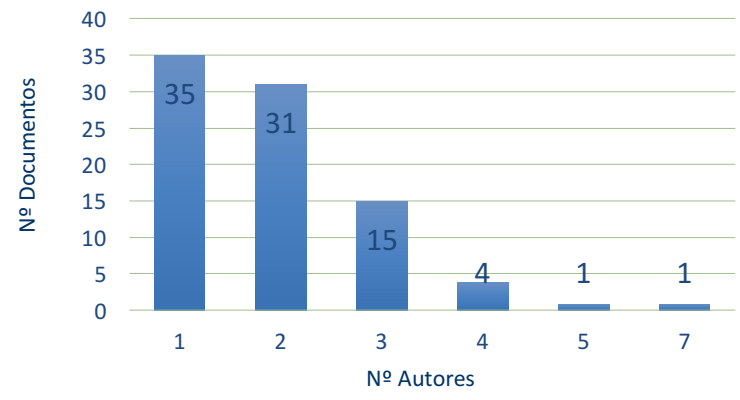

Gráfico 7. Número de autores por documento

La falta de coautoría se traduce en falta de relaciones interinstitucionales, y, por supuesto, internacionales, lo que supone un empobrecimiento en su visualización.

\begin{tabular}{|c|c|}
\hline Institución & $N .^{\circ}$ \\
\hline Universidad de Extremadura & 23 \\
\hline Corporación de Radio y Televisión Española & 6 \\
\hline Universidad Complutense de Madrid & 6 \\
\hline Universidad de Barcelona & 5 \\
\hline Universidad Autónoma de Barcelona & 4 \\
\hline Ghent University & 3 \\
\hline Netherlands Institute for Sound and Vision & 3 \\
\hline Archivo Histórico de Sabadell & 2 \\
\hline Radiotelevisión Valenciana & 2 \\
\hline Universidad Central de Ecuador & 2 \\
\hline Universidad del País Vasco & 2 \\
\hline Universidad Oberta de Catalunya & 2 \\
\hline Universidad Ramón Llull & 2 \\
\hline Université de Montréal & 2 \\
\hline Accenture Outsourcing Services S.A. & 1 \\
\hline Antena 3 TV S.A. & 1 \\
\hline BBC Research \& Innovation & 1 \\
\hline Caisse de dépôt et placement du Québec & 1 \\
\hline Canal Extremadura & 1 \\
\hline City University of London & 1 \\
\hline Copenhagen Business School & 1 \\
\hline German Broadcasting Architecture & 1 \\
\hline German National Library of Science and Technology & 1 \\
\hline Gnuo Consultores & 1 \\
\hline Icelandic Radiation Safety Authority & 1 \\
\hline Istituto di Scienza e Tecnologie dell'Informazione & 1 \\
\hline Lebanese University & 1 \\
\hline Leibniz Universität Hannover & 1 \\
\hline Loyola University Maryland & 1 \\
\hline Madeira Interactive Technologies Institute & 1 \\
\hline National Technical University of Athens & 1 \\
\hline New York University & 1 \\
\hline
\end{tabular}

\begin{tabular}{ll}
\hline Otra Pantalla & 1 \\
\hline Penn State University & 1 \\
\hline Punjabi University & 1 \\
\hline Pusan National University & 1 \\
\hline Radiotelevisión del Principado de Asturias & 1 \\
\hline Technische Universität Braunschweig & 1 \\
\hline Televisió de Catalunya & 1 \\
\hline UiT The Arctic University of Norway & 1 \\
\hline Universidad Camilo José Cela & 1 \\
\hline Universidad de Costa Rica & 1 \\
\hline Universidad de Salamanca & 1 \\
\hline Universidad Federal do Estado do Rio de Janeiro & 1 \\
\hline Universidad Miguel Hernández & 1 \\
\hline Universidad Nacional Autónoma de México & 1 \\
\hline Universidad Nacional de Educación a Distancia & 1 \\
\hline Universidad Politécnica de Valencia & 1 \\
\hline Universidad Pompeu Fabra & 1 \\
\hline Universidad Rey Juan Carlos & 1 \\
\hline Universidade do Porto & 1 \\
\hline Universidade Federal do Rio de Janeiro & 1 \\
\hline Universität Marburg & 1 \\
\hline Universiteit Twente & 1 \\
\hline University of Amsterdam & 1 \\
\hline University of Botswana & 1 \\
\hline University of Leicester & 1 \\
\hline University of London & 1 \\
\hline University of Massachusetts & 1 \\
\hline University of Oklahoma & 1 \\
\hline University of Patras & 1 \\
\hline University of Sheffield & 1 \\
\hline University of South Africa & 1 \\
\hline Utrecht University & 1 \\
\hline Vrije Universiteit Amsterdam & 1 \\
\hline Washington State University Libraries & 1 \\
\hline WGBH Boston & 1 \\
\hline WNET TV New York City & 1 \\
\hline Yeungnam University & 1 \\
\hline & 1 \\
\hline
\end{tabular}

Tabla II. Número de documentos por institución

El análisis de las instituciones (Tabla II) aporta nuevos datos reveladores: la preponderancia de las universidades españolas y la presencia de autores de la cadena pública Televisión Española. Sale destacada la Universidad de Extremadura, aunque existe un importante número de trabajos por parte de la Universidad Complutense de Madrid, Universidad de Barcelona y Universidad Autónoma de Barcelona. Seguida de ellas se encuentran Ghent University y el Netherlands Institute for Sound and Vision. Junto a Televisión Española, la entidad holandesa señalada, son las únicas que destacan fueran del ámbito de la academia.

Entre las instituciones con un menor número de trabajos (dos o menos) las principales aportaciones cuantitativamente vienen de la mano del Archivo Histórico de Sabadell, Radiotelevisión archivos televisivos en la Web of Science (2000-2016). // Ibersid. 11:2 (jul.-dic. 2017) 73-80. ISSN 1888-0967. 
Valenciana, Universidad Central de Ecuador, Universidad del País Vasco, Universidad Oberta de Catalunya, Universidad Ramón Llull y la Universidad de Montreal.

Entre otras instituciones se encuentran cadenas de televisión, tanto españolas como extranjeras, empresas, archivos, etc. Los autores afiliados a dichas instituciones, y firmantes de un mínimo de dos trabajos, son los que se muestran a continuación (Tabla III).

\begin{tabular}{lc}
\hline Autor & $N^{\circ}$ \\
\hline Caldera-Serrano J & 22 \\
\hline López-De-Solís I & 4 \\
\hline Guallar J & 3 \\
\hline Sánchez-Jiménez R & 3 \\
\hline Agirreazaldegi-Berriozábal T & 2 \\
\hline De Sutter R & 2 \\
\hline Fernández-Quijada D & 2 \\
\hline Freire-Andino Ro & 2 \\
\hline González-Ruiz D & 2 \\
\hline Gunter B & 2 \\
\hline Hauttekeete L & 2 \\
\hline León-Moreno JA & 2 \\
\hline López-De-Quintana-Sáenz E & 2 \\
\hline Notebaert S & 2 \\
\hline Ribera M & 2 \\
\hline Termens M & 2 \\
\hline Turner Jm & 2 \\
\hline Van De Walle R & 2 \\
\hline Zapico-Alonso F & 2 \\
\hline
\end{tabular}

Tabla III. Autores con más de dos publicaciones

A pesar de que en la tabla anterior no se muestren los autores firmantes de un solo documento, es digno de mencionar que esta cifra supera los 100 , dato muy elevado para tan escaso número de trabajos. Dudamos de si realmente estos autores son expertos en documentación audiovisual o si sencillamente no aplican a revistas presente en la WoS, elemento que queremos destacar posteriormente en las conclusiones. Vuelven a destacar los autores españoles, que corresponden obviamente con las instituciones que anteriormente se indicaron como las más productivas.

\section{Conclusiones}

A la vista de los resultados del presente estudio, $\mathrm{y}$ de las potenciales lecturas que facilitan cada uno de los gráficos y tablas presentados, se entiende en primer lugar que existe un número escaso de publicaciones de una especialización de las Ciencias de la Documentación que lleva muchos años desarrollándose. No obstante, muchos de estos trabajos no están presentes en la WoS, por lo que podemos dar varias lecturas a este hecho.

La primera de ellas es que las revistas presentes en la WoS no tienen entre sus líneas de investigación los archivos televisivos, pudiendo publicar en aquellas de carácter más generalista.

La segunda de las posibles causas es la falta de interés por parte de los autores de publicar en la WoS para internacionalizar sus resultados. Seguramente retroalimentada con la primera causa, hace que los trabajos sean publicados en revistas locales y no se tenga en cuenta la publicación a un nivel superior.

Una tercera causa es la localización mayoritaria de los autores en España y la publicación en revista de habla hispana. La producción científica difundida en español tiende a tener menor visibilidad y a tener menos potencialidad de ser publicado, simplemente porque el número de revistas presente en la WoS que publican trabajos en español es poco significativo.

Además del escaso número de resultados obtenidos, debe quedar reflejado la clara presencia de autores y revistas del ámbito hispano, destacando la revista El Profesional de la Información por su apertura a publicar este tipo de temáticas, tanto en monográficos como a lo largo de sus números. La relación y la publicación de trabajos de Comunicación, hace a esta publicación como el eslabón más relevante entre las dos disciplinas (Documentación y Comunicación), donde la gestión de la información en los medios de comunicación es la máxima relación entre las dos áreas.

No obstante, observamos una tendencia en el aumento del número de citas, que puede venir derivado de la publicación cada vez mayor de trabajos en inglés (por parte de todos los autores), al incremento del número de publicaciones (cercano a los diez en los últimos años) y a la inclusión de nuevas revistas procedentes de la base de datos creadas por WoS ESCI.

El escaso nivel de coautoría se plantea como un grave inconveniente para una mayor visualización. Coautoría que debe potenciarse, especialmente a nivel internacional. Es de agradecer la coautoría entre autores procedentes de cadenas televisivas y de la universidad, acercando la visión de la academia y la empresa. No obstante, es importante el número de autores que publican en solitario, con fórmulas más cercanas a las Humanidades que a las Ciencias Sociales.

\section{Reconocimientos}

Este trabajo ha sido financiado por el Gobierno de Extremadura (Consejería de Educación, Ciencia y Tecnología) y el 
Fondo Social Europeo dentro del plan de apoyo a las actuaciones de los Grupos de Investigación inscritos en el catálogo de la Junta de Extremadura. GR10019.

\section{Referencias}

Agirreazaldegi-Berriozabal, Teresa (2007). Claves y retos de la documentación digital en televisión. // El Profesional de la Información. 16:5, 433-442.

Bustos-Pérez-de-Salcedo, Pilar-de (2007). Sistemas integrados y gestión documental. La experiencia en Telecinco. // El Profesional de la Información. 16:5, 450-455.

Caldera-Serrano, Jorge (2008). Changes in the management of Information in audio-visual archives following digitization: current and future Outlook. // Journal of Librarianship and Information Science. 40:1, 13-20.

Caldera-Serrano, Jorge; Arranz-Escacha, Pilar (2012). Documentación audiovisual en televisión. Barcelona: Editorial UOC, 2012. Colección El profesional de la información; 13.

Castillo Blasco, Lourdes; Soler Monreal, Concha (2014). Análisis de los artículos publicados sobre documentación televisiva en España (1984-2014). // BiD: textos universitaris de bibliotecomia y documentació. 33, 1-13 http://bid.ub.edu/es/33/castillo2.htm

De la Cuadra, Elena; López de Solís, Iris (2012). Imágenes de archivo en cine de ficción: cine basado en una historia real. Trípodos. 31: 11-36

Edmondson, Ray (2004). Filosofía y principios de los archivos audiovisuales. París: Unesco.

Giménez Rayo, Mabel (2007). Documentación audiovisual de televisión: la selección del material. Gijón: Trea.

Hidalgo Goyanes, Paloma (2005). La documentación audiovisual de las televisiones: la problemática actual y el reto de la digitalización. // Documentación de las Ciencias de la Información. 28, 159-171.
Hidalgo Goyanes, Paloma (2013). Patrimonio audiovisual en televisión. // Marcos Recio, Juan Carlos, coord. Gestión del patrimonio audiovisual en medios de comunicación. Madrid: Síntesis. 53-82.

Inarejos, Lluís; Guallar, Javier (2015). Centros de documentación de televisiones en Catalunya. Estudio de BTV, RTVE, TVC y 8tv. // Cuadernos de Documentación Multimedia. 26, 48-65

López-de-Quintana-Sáenz, Eugenio (2007). Transición y tendencias de la documentación en televisión: digitalización y nuevo mercado audiovisual. // El Profesional de la Información. 16:5, 397-408.

López-de-Quintana-Sáenz, Eugenio (2014). Rasgos y trayectorias de la documentación audiovisual: logros, retos y quimeras. // El Profesional de la Información. 23:1, 5-12.

López Yepes, Alfonso (1992). Los centros de documentación cinematográfica: panorama actual. // Documentación de las Ciencias de la Información. 15, 219-258.

López Yepes, Alfonso (2014). Patrimonio fílmico informativo: hacia una red temática de acceso abierto y proyección iberoamericana (Cinedocnet). // Ibersid: revista de sistemas de información y documentación. 8: 81-90.

Póveda-López, Inés-Carmen; Caldera-Serrano, Jorge; PoloCarrión, Juan-Antonio (2010). Definición del objeto de trabajo y conceptualización de los Sistemas de Información Audiovisual de la Televisión. // Investigación Bibliotecológica. 24:50, 15-34

Saavedra Bendito, Pau (2011). Los documentos audiovisuales. Qué son y cómo se tratan. Gijón: Trea.

Unesco (1980). Recomendación sobre la salvaguardia y la conservación de las imágenes en movimiento de la UNESCO. Aprobada por la Conferencia General en su $21^{1}$ reunión, Belgrado, 27 de octubre de 1980.

Enviado: 2017-03-10. Segunda versión: 2017-09-12. Aceptado: 2017-09-12 